\title{
LEYENDO PARA APRENDER: ENTENDIENDO LECTURAS CON ENSEÑANZA DE VOCABULARIO INTEGRADO EN ESTUDIANTES A1 DE INGLÉS COMO IDIOMA EXTRANJERO
}

\author{
"READING TO LEARN: UNDERSTANDING READING WITHIN \\ INTEGRATED VOCABULARY INSTRUCTION IN A1 EFL LEARNERS"
}

\author{
Gabriela Galeas Arboleda \\ UEM Carmelina Granja Villanueva, San Carlos. Los Ríos, Ecuador. \\ gabrielagaleas@hotmail.com
}

\section{RESUMEN}

El presente estudio cuantitativo experimental investigó los efectos de instrucción de vocabulario integrado en el desempeño de la comprensión lectora de estudiantes con nivel A1 de inglés como idioma extranjero en un colegio público rural en Ecuador. Los participantes fueron estudiantes entre 15 y 17 años en su primer año de bachillerato, todos ellos fueron seleccionados por un muestreo a conveniencia. Los participantes estuvieron divididos en dos grupos (tradicional e integrado) y se les solicitó participar en ocho lecciones de lectura. Después de cada clase, los estudiantes participaron en una prueba de comprensión lectora basada en tareas de comprensión literal. Además, las percepciones del grupo experimental fueron analizadas mediante un cuestionario. Adicionalmente, se utilizó una escala de conocimientos de vocabulario (VKS) tres veces, para medir el conocimiento previo, el aprendizaje y la retención de palabras. Este estudio muestra que la instrucción de vocabulario integrado mejora la comprensión de lectura de los estudiantes, y es beneficiosa para el aprendizaje y retención de vocabulario. Por otra parte, este trabajo de investigación propone un estudio adicional sobre los beneficios de una instrucción integrada al trabajar con niveles superiores de competencia.

Palabras claves: lectura, instrucción de vocabulario, comprensión lectora, percepciones.

\begin{abstract}
This experimental quantitative study explored the effects of an integrated vocabulary instruction on reading comprehension performance of A1 EFL learners in a rural public school in Ecuador. The participants of this study were students between 15 and 17 years old in their first baccalaureate year, all of them were selected by convenience sampling. The participants were divided into two groups (traditional and integrated) and asked to participate in eight reading lessons with their designated vocabulary instruction. After each class, they took part in a reading comprehension test based on literal comprehension questions. Moreover, the perceptions from the experimental group were gathered by a questionnaire. Additionally, a vocabulary knowledge scale (VKS) was used three times to measure previous knowledge, learning, and retention of words. This study shows that an integrated vocabulary instruction improves learners' reading comprehension, and it is beneficial for vocabulary learning and retention. Furthermore, this research work proposes a further study on the benefits of an integrated instruction by working with upper levels of proficiency.
\end{abstract}

Keywords: reading, vocabulary instruction, reading comprehension, perceptions.

Recibido: 24 de febrero de 2018

Aprobado: 07 de mayo de 2018

Publicado: 20 de junio de 2018 


\section{Introducción}

Nation (2009) ha mencionado que dos de los enfoques de lectura intensiva son comprensión la cual busca el interpretar un texto en particular y vocabulario que se enfoca en el aprendizaje de palabras (significado y uso). Además, el manejo de un adecuado léxico es primordial para la comunicación y un prerrequisito para la comprensión lectora. Chall (1987 citado en Naimi \& Chow, 2015) mostró que un adecuado conocimiento de palabras contribuye a la comprensión lectora, y que las habilidades de lectura pueden promover el aprendizaje de vocabulario.

Una revisión realizada por Hamzehlou, Zainal and Ghaderpour (2012) sobre la importancia de vocabulario en la adquisición del idioma permite entender la intricada relación entre vocabulario y comprensión lectora. Por ejemplo, los estudiantes pueden experimentar dificultades con palabras desconocidas mientras están leyendo. En cuanto a esta dificultad, profesores y estudiantes concuerdan que uno de los obstáculos más significativos en la comprensión de un texto es el reconocimiento de palabras o el acceso léxico. Sin embargo, muchos profesores de inglés como idioma extranjero (EFL) desafían la capacidad de sus estudiantes para lidiar con palabras desconocidas en un texto. Asimismo, existe una consideración significativa de que la profundidad y la amplitud del conocimiento del léxico y la comprensión de la lectura están intrincadamente relacionados entre sí (Hamzehlou, Zainal, \& Ghaderpour, 2012).

La comprensión lectora es considerada el propósito central al leer, pero en algunas ocasiones es considerada frustrante o confusa. En otras palabras, la comprensión lectora puede llegar a ser complicada cuando se incluye su propósito, propiedades y la lectura en sí para la enseñanza. Algunos estudios lo demuestran cuando se enfocan en el efecto del tamaño de vocabulario sobre la comprensión lectora (Hamzehlou, Zainal, \& Ghaderpour, 2012) o las consecuencias de comprensión de lectura sobre el tamaño de vocabulario (Gungor \& Yayh, 2016).

Debido a que la literatura propone la lectura y el aprendizaje de vocabulario como actividades simultáneas (Bachowickz, Fisher, \& Ogle, 2006) el propósito de esta investigación es describir los efectos de la enseñanza de vocabulario integrado (IVI) en las actividades de comprensión lectora al examinar el desempeño de estudiantes $A 1$ de inglés como idioma extranjero en primero bachillerato.

\section{Preguntas de Investigación}

El presente estudio de investigación pretendió responder la siguiente pregunta principal:

¿Cómo la enseñanza de vocabulario integrado promueve el rendimiento de la comprensión lectora de los estudiantes $A 1$ de inglés como idioma extranjero de primero bachillerato de un colegio público?, al mismo tiempo buscó responder las siguientes sub-preguntas:

1. ¿Existe una variación en el rendimiento de la comprensión de lectura con respecto a un grupo experimental y de control de estudiantes A1 de inglés como idioma extranjero de primero bachillerato de un colegio público?

2. ¿Cuáles son las percepciones de los estudiantes del grupo experimental hacia la enseñanza de vocabulario integrado?

3. ¿La instrucción del vocabulario integrado es significativamente beneficiosa para la retención de vocabulario?

\section{Materiales y métodos}

El presente estudio buscó comparar los resultados entre un grupo de control y experimental, por lo tanto, se encuentra alineado a un diseño experimental con una metodología cuantitativa. El investigador empleó un cuasi experimento que permitió tomar grupos intactos de una población en particular sin asignación al azar de los sujetos. En el momento inicial del estudio, hubo un total de $(\mathrm{N}=82)$ participantes, 37 hombres jóvenes y 45 mujeres jóvenes con edades entre 15 a 18 años. Sin embargo, en la etapa final, hubo 66 estudiantes, 35 participantes en el grupo de control y 31 en el grupo experimental. Los participantes en ambos grupos pertenecían a la jornada vespertina y se encontraban cursando el primer año bachillerato con nivel A1 de acuerdo con el Marco Común Europeo de Referencia (CEFR).

La presente investigación empleó tres instrumentos que permitieron al investigador recolectar los datos necesarios para responder las preguntas de investigación propuestas. Los tests de lecturas permitieron medir el rendimiento de comprensión, una escala de conocimiento de glosario sirvió para determinar el conocimiento de palabras especificas en tres etapas del estudio, y finalmente, un cuestionario recogió las percepciones del grupo experimental sobre la enseñanza de vocabulario integrado. Las sesiones de lectura se efectuaron por cuatro semanas en el horario regular de los estudiantes, dos sesiones por semana. 
En el grupo de control, los participantes aprendieron el vocabulario específico de forma tradicional, es decir, los participantes estudiaron las palabras desconocidas antes de ser expuestos a las lecturas. Cada clase empezó con una actividad previa relacionada al texto. Seguido de esto, el profesor presentó las palabras desconocidas usando imágenes, dando énfasis a la forma con la que sería presentada en la lectura y formando oraciones. Luego de esto, se realizó una discusión con el título de la lectura o imágenes disponibles para predecir el contenido de la lectura. Finalmente, todos los estudiantes participaron en la lectura con la ayuda del profesor quien controlaba el ritmo de la lectura y garantizaba que todos los participantes pudieran procesar el texto.

En un segundo escenario, pero con una diferencia significativa, el grupo experimental empezaba la clase con una actividad previa, luego se procedía a la discusión del título o imágenes de la lectura y así tener una idea del texto a ser explorado, después de esto, el investigador o un estudiante voluntario leía el texto mientras el resto de los estudiantes lo seguían de forma silenciosa. Al momento de encontrar una oración que incluía una palabra desconocida, el lector se detenía al final de la oración, dando lugar para que el investigador pueda mostrar la palabra a través de una imagen, dando un sinónimo en inglés o su traducción, definiendo su rol en la oración y realizando una nueva con la ayuda de los estudiantes. En este proceso, las palabras desconocidas fueron presentadas en el contenido del texto. El investigador tomó en consideración el tiempo asignado para la enseñanza de vocabulario en cada grupo y así evitó una exposición extra en clase. Otro punto clave es que los estudiantes pudieron tomar notas como usualmente lo hacen en sus clases tradicionales con el fin de prevenir desacuerdos en el ambiente tradicional de enseñanza/aprendizaje.

Luego de cada clase, los participantes de ambos grupos participaron de un test de compresión lectora que permitió determinar diferencias en el desempeño lector. Cada test estaba compuesto de dos partes: la primera parte, basada en preguntas pronominales o de si/no, y la segunda parte con oraciones de completar. Las preguntas de compresión fueron diseñadas para ser de comprensión literal y motivar la lectura en los estudiantes mientras que las oraciones para ser completadas no fueron exactas al texto, sin embargo, las palabras faltantes se encontraban en la lectura. Además, no hubo letra inicial como ayuda en las oraciones. Cada test de compresión lectora contenía cinco preguntas y cinco oraciones incompletas.

Cada lectura y su test de comprensión fueron piloteados con 35 estudiantes no participantes del estudio, pero con características similares tanto en nivel de inglés, género y edad. Sin embargo, antes de ser usados, fueron revisados por un profesional de enseñanza del inglés como idioma extranjero, recibiendo sugerencias y haciendo algunas modificaciones a las preguntas. Después de esto, los test fueron contestados por un docente de la misma institución.

Como parte del estudio se usó y adaptó un cuestionario de la literatura para conocer las percepciones de los estudiantes que participaron el grupo de vocabulario integrado. Los ítems 1 al 5 permitieron conocer las percepciones relaciones a las actividades de comprensión (Guthrie and Lutz, 2014) y elementos 6-10 para conocer sus las percepciones sobre el aprendizaje de vocabulario con lecturas (Ali, Mukundan, Baki and Mohd, 2012). La confiabilidad del cuestionario fue comprobada con Cronbach's Alpha coefficients (Bryman, 2012), obteniendo un coeficiente de consistencia interna de 0.8. La administración de la encuesta se realizó un día después de la última lectura para permitir la asimilación de lo que habían aprendido en las clases de lectura y evitar confusión en como percibieron la instrucción.

Finalmente, se empleó una escala de conocimiento de vocabulario (Vocabulary Knowledge Scale) (Kim, 2011; Golonka, Bowles, Kramasz, Blake \& Buckwalter, 2015) para medir los primeros conocimientos de vocabulario, su post-adquisición, y retención de palabras. El primer VKS fue administrado una semana antes que el estudio comience, este permitió capturar los primeros niveles del conocimiento de palabras a ser presentadas durante el estudio. Una segunda administración se dio lugar después de la finalización del estudio para reportar el aprendizaje de palabras. Finalmente, una tercera administración tomo lugar dos semanas después de la finalización de las clases de lectura con el objetivo de medir el aprendizaje y retención de las palabras luego de haber asimilado la información. Cada test incluía 43 palabras.

\section{Resultados}

Los puntajes obtenidos de los tests de comprensión lectora fueron codificados en un programa estadístico PSS y analizados en 
independent sample t-tests. Los gráficos proporcionan un claro entendimiento de los hallazgos para comparar y conocer la diferencia sobre el rendimiento de comprensión lectora, los datos de las percepciones del grupo experimental y la información de los tres VKS. Gran parte de tablas y gráficos se obtuvieron de un SPSS software (PASW Statistic 18) (Bryman, 2012).

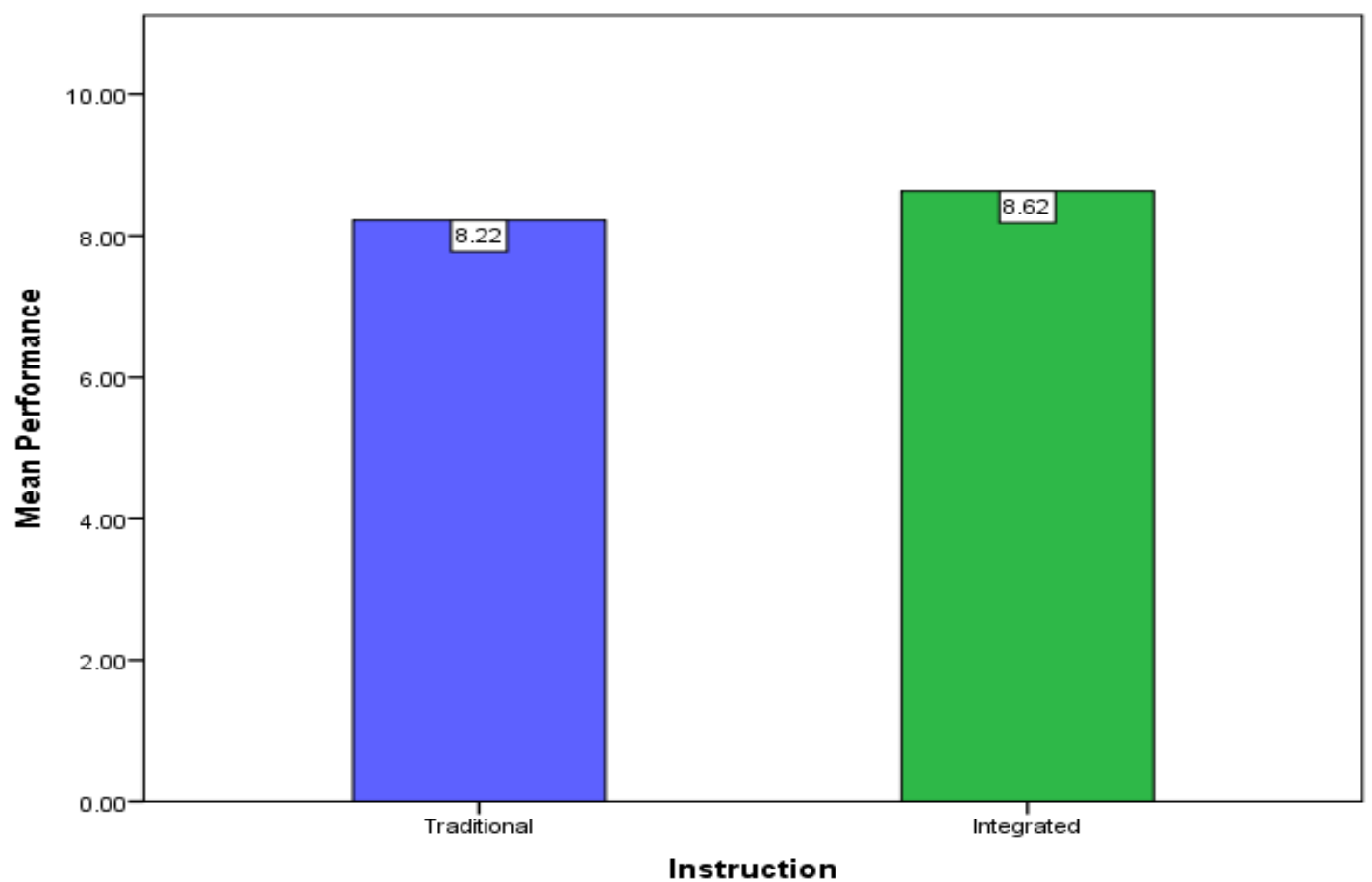

Figura 1. Media de las pruebas de comprensión lectora.

La figura muestra una variación entre los dos grupos, donde los estudiantes que participaron de vocabulario integrado probaron tener mejor desempeño en las tareas de comprensión lectora que aquellos estudiantes expuestos a la instrucción tradicional de enseñanza de vocabulario. Naeimi and Chow (2015) exponen que a medida que el conocimiento de vocabulario mejora, el desempeño de la comprensión lectora también lo hace.

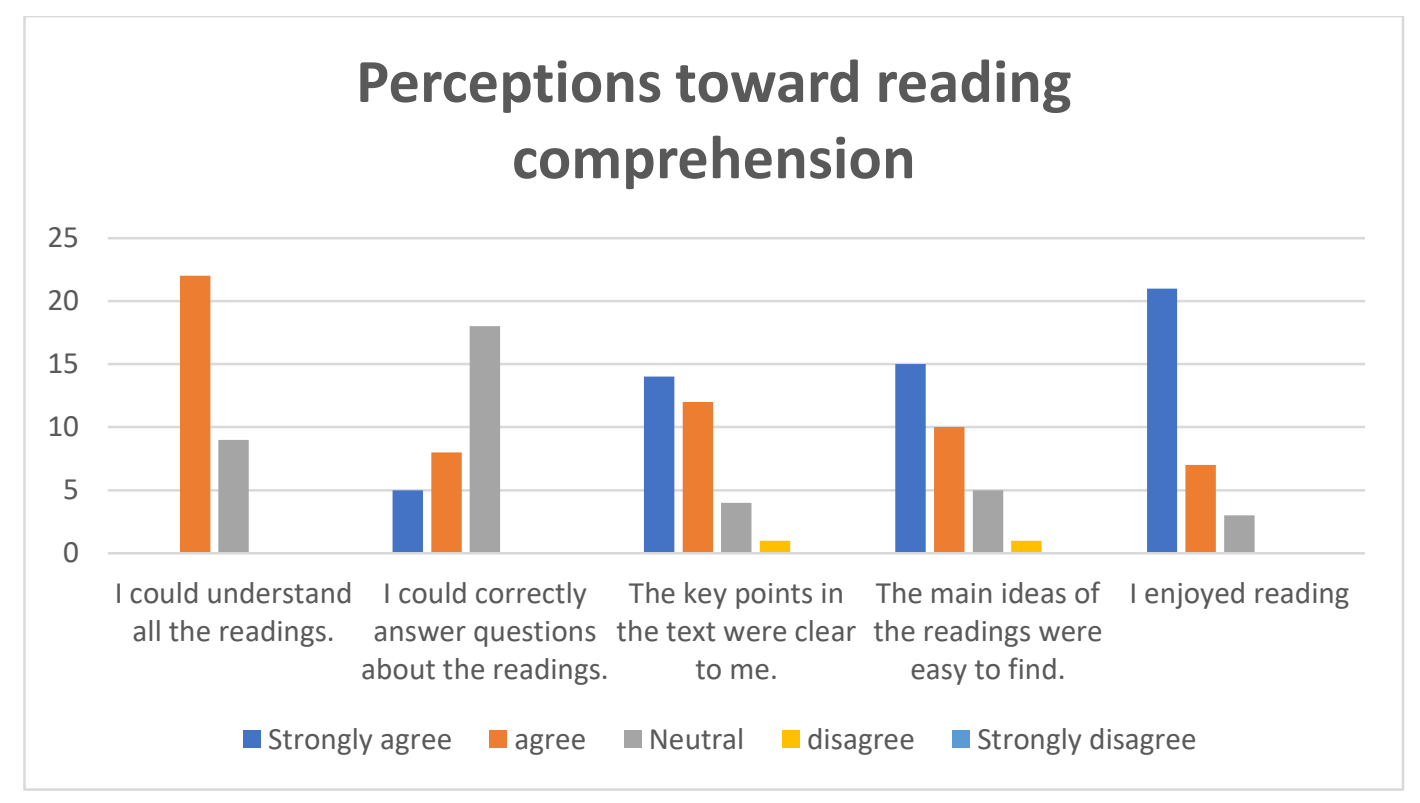

Figura 2. Percepción de comprensión lectora. 
Al hablar sobre la comprensión de lectura del grupo experimental durante el estudio, la mayoría de los estudiantes manifestaron que pudieron comprender los pasajes de lecturas, sin embargo, 18 de 31 se sintieron inseguros sí respondieron o no correctamente las tareas, esto es contradictorio porque una mayoría estuvo muy de acuerdo y de acuerdo en que los puntos clave y las ideas principales del texto fueron fácil de identificar.
Uno de los ítems más interesante sobre comprensión lectora corresponde al interés por las lecturas, aquí una gran mayoría de participantes mostraron un alto nivel de satisfacción. Como las lecturas eran adecuadas para el nivel de los estudiantes (Harmer, 2007) y los temas eran familiares, se sintieron motivados a participar en ellas.

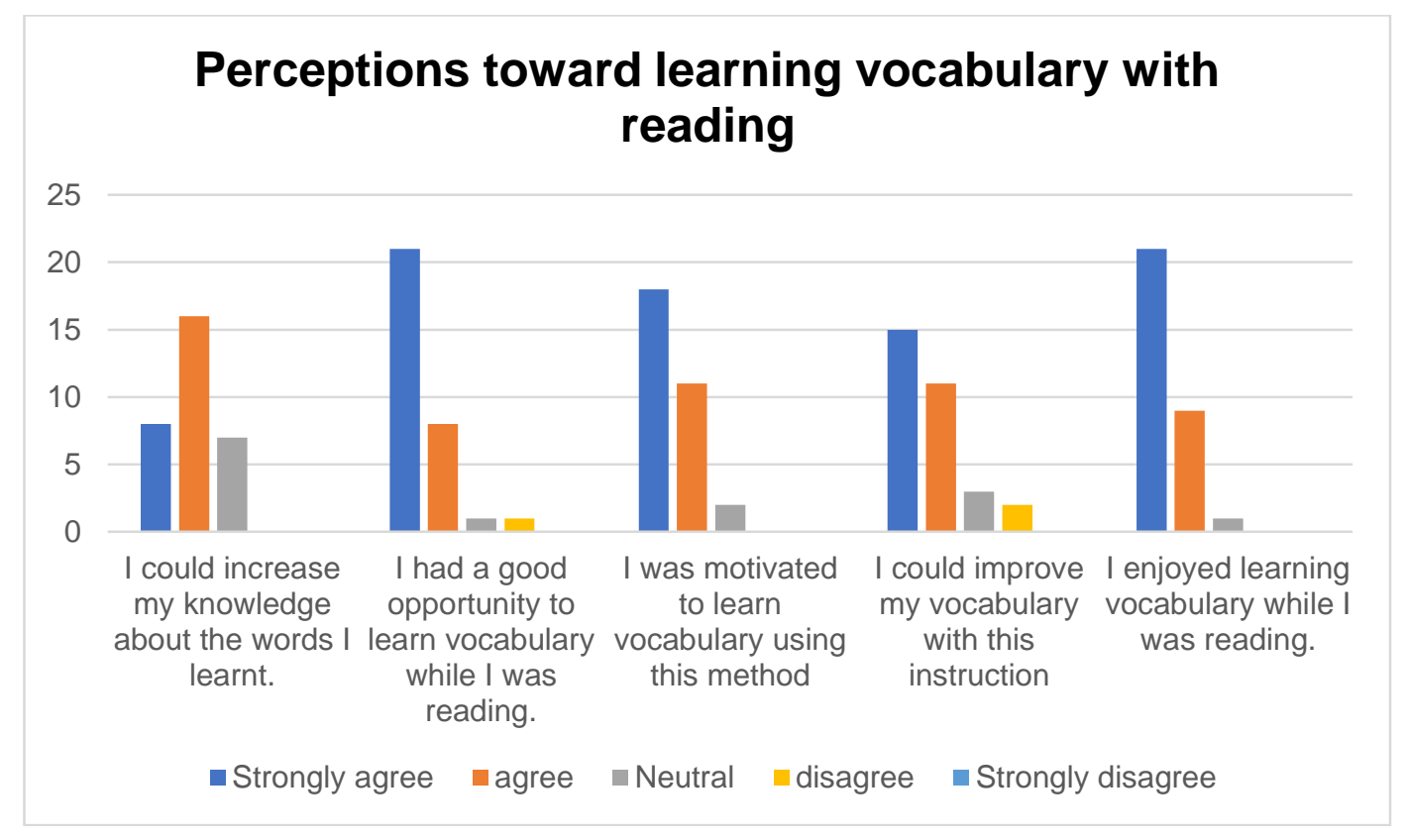

Figura 3. Percepción de aprendizaje de vocabulario con lectura.

La segunda parte del cuestionario en cuanto al aprendizaje de vocabulario con lecturas mostró que un gran porcentaje de estudiantes concordaron al percibir un incremento en el conocimiento de palabras. Además, la mayoría de los participantes $(n=21)$ estuvieron muy de acuerdo que la instrucción de vocabulario integrado es una buena oportunidad para aprender palabras mientras están leyendo. Nation (2009) también considero la lectura intensiva como una oportunidad de enfocarse en otras características del lenguaje como gramática o vocabulario.
Además, muchos de ellos se sintieron emocionados de aprender nuevas palabras y tenían una impresión de mejorar su conocimiento del vocabulario. Para ser más específico, 26 estudiantes coincidieron en sus percepciones entre de acuerdo y muy de acuerdo. Además, los participantes en el grupo de vocabulario integrado compartieron un total acuerdo al percibir una experiencia amena para aprender el vocabulario mientras exploraban los pasajes. 


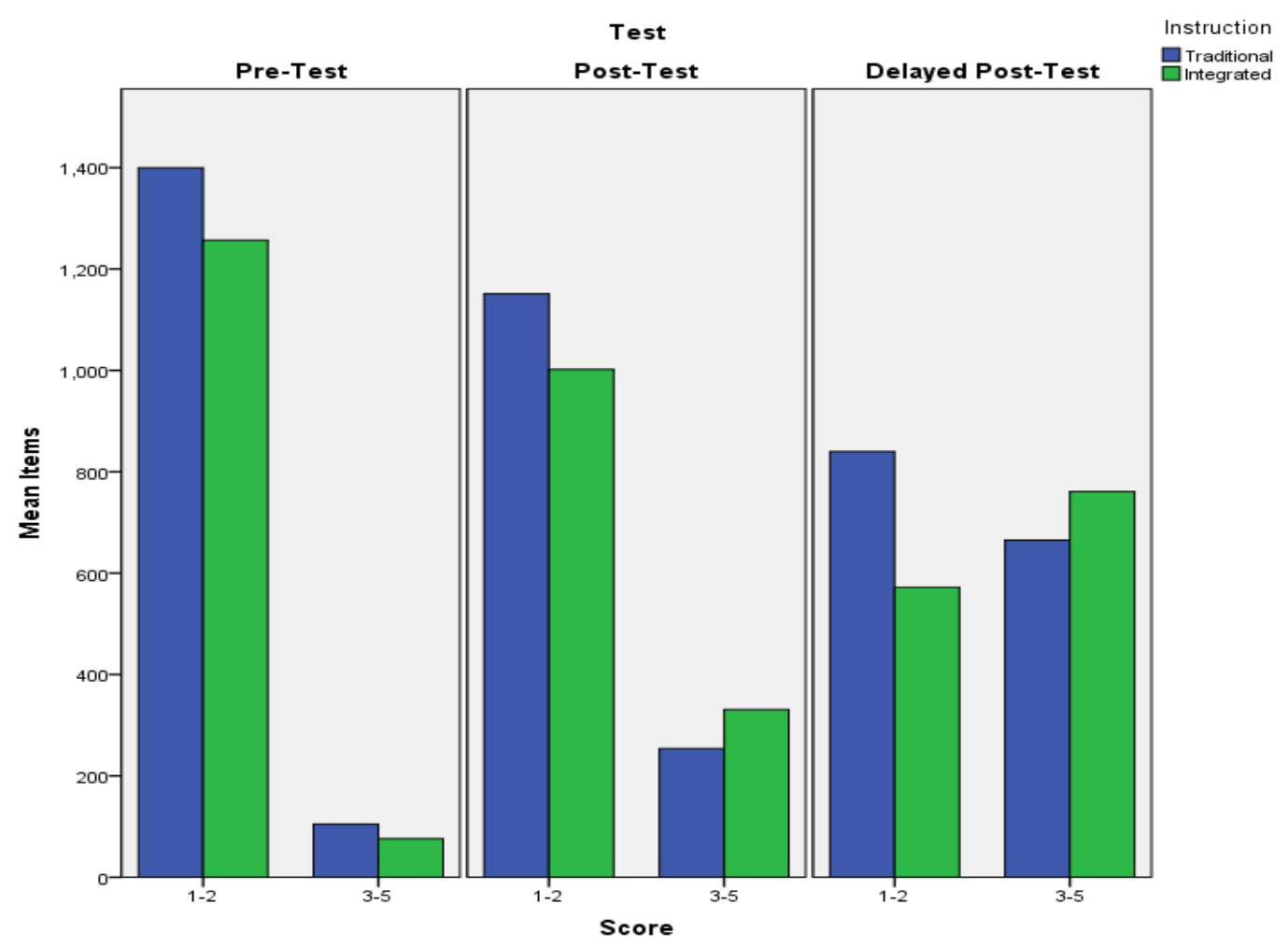

Figura 4. Comparación de los puntajes obtenidos en la escala de conocimiento de vocabulario (VKS).

Los resultados de este estudio indicaron que ambos tipos de enseñanza (tradicional e integrado) promueven un alto aprendizaje y retención de palabras. El gráfico muestra que 1 y 2 representa un mínimo de conocimiento o nada de las palabras y 3-5 representa que las han visto, las conocen o saben usarla. Del mismo modo el total de palabras es multiplicado por la cantidad de participantes $(43 * 35$ en el grupo de control y 31 en el experimental) y así se obtiene los puntajes: Estos hallazgos son apoyados por Andrew and Adams (2010) cuando mostraron sus resultados en cuanto a retención de vocabulario; ellos concluyeron que ambos (ya sea aislados o integrado) conducen a una mayor retención y aprendizaje de conocimientos de palabras. Sin embargo, Schmitt, Jian, and Grabe (2011) expresaron que cuando existe una palabra desconocida, los estudiantes se esfuerzan por entender tanto como les es posible o buscando su significado en el texto si aparece numerosas veces y así entender lo que está leyendo.

Además, cuando una palabra desconocida aparece en un texto, los estudiantes pueden usar pistas como el significado de la oración, la morfología de la palabra y el significado del discurso (Yin, 2013). En otras palabras, hay logros significativos en el uso de una instrucción para enseñar palabras en el contenido de un texto porque a pesar de que requiere un esfuerzo extra, estudiantes pueden aumentar su retención y aprendizaje.

\section{Conclusiones}

El presente estudio demostró que los estudiantes que experimentaron la enseñanza de vocabulario integrado tuvieron mejor desempeño en su comprensión lectora que aquellos estudiantes expuestos a la enseñanza tradicional. Por lo tanto, se sugiere enseñar el significado, el uso y la forma de las palabras desconocidas a lo largo de la lectura para promover su comprensión (IVI), especialmente con estudiantes $\mathrm{A} 1$.

Además, la percepción de los estudiantes en el grupo experimental reveló una comodidad y familiaridad en lecciones de lectura intensiva con instrucción de vocabulario integrado, ya que les permite aprender nuevas palabras sin restricción mientras están leyendo y a su vez comprender y disfrutar la lectura.

Asimismo, la enseñanza integrada promueve un objetivo dual (comprensión de lectura y retención de vocabulario) por lo que se recomienda su implementación en lectura intensiva. Sin embargo, debido a que tanto la instrucción tradicional aislada o integrada de vocabulario fomentan su aprendizaje y retención, el instructor puede elegir la instrucción integrada o aislada dependiendo de 
las necesidades del alumno/profesor o estilo de aprendizaje.

En cuanto a las limitaciones, debido a las complicaciones para asistir a un horario extracurricular, los participantes estaban en el mismo grupo con el nivel y la edad de otros estudiantes en sus clases regulares. Otra limitación fue el tiempo, un estudio más extenso podría exponer una mejor visión de los resultados con respecto a la comprensión lectora y la retención de palabras en la enseñanza de vocabulario integrada.

Finalmente, este estudio propone explorar los efectos de enseñanza de vocabulario integrada con niveles más altos de competencia lingüística y pruebas de comprensión superiores a la comprensión literal.

\section{Referencias}

1. Ali, Z., Mukundan, J., Baki, R., \& Mohd, A. (2012). Second Language Learners' Attitudes towards the Methods of Learning Vocabulary. English Language Teaching, 5(4), 24-36. doi:10.5539/elt.v5n4p24

2. Andrew, K., \& Adams, R. (2010). Should Vocabulary Instruction Be Integrated or Isolated? TESOL Quarterly, 44(2), 222249. doi:10.5054/tq.2010.219943

3. Bachowickz, C., Fisher, P., \& Ogle, D. (2006). Vocabulary: Questions from the classroom. Reading Research Quarterly, 41(4), 524-539. doi:10.1598/RRQ.41.4.5

4. Bryman, A. (2012). Social Research Methods (4th ed.). New York, NY: Oxford University Press.

5. Golonka, E., Bowles, A., Silbert, N., Kramasz, D., Blake, C., \& Buckwalter, T. (2015). The Role of Context and Cognitive Effort in Vocabulary Learning: A Study of Intermediate-Level Learners of Arabic. The Modern Language Journal, 99(1), 19-39. doi: $10.1111 /$ modl.12191
6. Gungor, F., \& Yayh, D. (2016). The Interplay between Text-based Vocabulary Size and Reading Comprehension of Turkish EFL Learners. Educational Sciences: Theory and Practice, 16(4), 1171-1188. doi:10.12738/estp.2016.4.0078

7. Guthrie, J., \& Lutz, S. (2014). Effects of Classroom Practices on Reading Comprehension, Engagement, and Motivations for Adolescents. Reading Research Quarterly, 49(4), 387-416. doi:10.1002/rrq.81

8. Hamzehlou, S., Zainal, Z., \& Ghaderpour, M. (2012). A Review on the Important Role of Vocabulary Knowledge in Reading Comprehension Performance. Procedia: Social and Behavioral Sciences, 66, 555563. doi:10.1016/j.sbspro.2012.11.300

9. Harmer, J. (2007). How to Teach English. Harlow, England: Pearson Education Limited.

10. Kim, Y. (2011). The Role of Task-Induced Involvement and Learner Proficiency in L2 Vocabulary Acquisition. Language Learning, 61(Suppl.1), 100-140. doi: 10.1111/j.1467-9922.2011.00644.x

11. Nation, P. (2009). Teaching ESL/EFL Reading and Writing. New York: Routledge.

12. Naeimi, M., \& Chow, T. (2015). Vocabulary Acquisition through Direct and Indirect Learning Strategies. English Language Teaching, $\quad 8(10), \quad 142-151$. doi:10.5539/elt.v8n10p142

13. Schmitt, N., Jiang, X., \& Grabe, W. (2011). The Percentage of Words Known in a Text and Reading Comprehension. The Modern Language Journal, 95(i), 26-43. doi:10.1111/j.1540-4781.2011.01146.x

14. Yin, Z. (2013). Infer the Meaning of Unknown Words by Sheer Guess or by Clues?- An Exploration on the Clue Use in Chinese EFL Learner's Lexical Inferencing. English Language Teaching, 6(11). 